\title{
The new Tunka-133 EAS Cherenkov array: Status of 2009
}

\author{
B.V. Antokhonov ${ }^{\text {a }}$, S.F. Beregnev ${ }^{a}$, N.M. Budnev ${ }^{\text {b }}$, O.B. Chvalaev ${ }^{b}$, A. Chiavassa ${ }^{\text {, O.A. Gress }}{ }^{\text {b,* }}$, \\ N.N. Kalmykov ${ }^{a}$, N.N. Karpov ${ }^{\text {a }}$, E.E. Korosteleva ${ }^{\text {a }}$, V.A. Kozhin ${ }^{\text {a }}$, L.A. Kuzmichev ${ }^{\text {a }}$, B.K. Lubsandorzhiev ${ }^{c}$, \\ R.R. Mirgazov ${ }^{\text {}}$, M.I. Panasyuk ${ }^{a}$, L.V. Pankov ${ }^{b}$, V.V. Prosin ${ }^{a}$, V.S. Ptuskin ${ }^{d}$, Yu.A. Semeney ${ }^{b}$, \\ B. Shaibonov Jr. ${ }^{c}$, A.A. Silaev ${ }^{a}$, A.A. Silaev Jr. ${ }^{a}$, A.V. Skurikhin ${ }^{a}$, Ch. Spiering ${ }^{\mathrm{e}}$, R. Wischnewski ${ }^{\mathrm{e}}$, I.V. Yashin $^{\mathrm{a}}$, \\ A.V. Zablotsky ${ }^{\text {, }}$, A.V. Zagorodnikov ${ }^{\text {b }}$
}

\footnotetext{
a Skobeltsyn Institute of Nuclear Physics of Moscow State University, Moscow 119991, Russia

${ }^{\mathrm{b}}$ Institute of Applied Physics of Irkutsk State University, Irkutsk 664003, Russia

${ }^{\mathrm{c}}$ Institute for Nuclear Research of Russian Academy of Sciences, Moscow 117312, Russia

${ }^{\mathrm{d}}$ Institute of Terrestrial Magnetism, Ionosphere and Radiowave Propagation of Russian Academy of Sciences (IZMIRAN), Troitsk, Moscow Region 142092, Russia

e Deutsches Elektronen Synchrotron (DESY-Zeuthen), Zeuthen D-15738, Germany

${ }^{\mathrm{f}}$ Torino University, Via P.Giuria, 1, 10125 Torino, Italy
}

\section{A R T I C L E I N F O}

Available online 11 November 2010

Keywords:

Cosmic rays

Extensive air shower

Cherenkov light

Data acquisition system

\begin{abstract}
A B S T R A C T
The deployment of the new Extensive air shower Cherenkov installation Tunka-133 with about $1 \mathrm{~km}^{2}$ geometric acceptance area was completed in October 2009. The array will permit a detailed long-term study of the cosmic ray energy spectrum and mass composition in the energy range $10^{15}-10^{18} \mathrm{eV}$ with a unique and more elaborate method. The array construction and data acquisition system, preliminary results and plans for future development are presented.
\end{abstract}

(c) 2010 Elsevier B.V. All rights reserved.

\section{Introduction}

The detailed study of the cosmic ray energy spectrum and primary mass composition in the energy range $10^{15}-10^{18} \mathrm{eV}$ is of crucial importance for the understanding of the origin and propagation of cosmic rays in the Galaxy. Even after several decades of study, there is no clear understanding of the origin of the knee and second knee. Clearly data obtained with higher sensitivity and less uncertainty in the estimation of primary energy and composition are expected to provide a better understanding of these important features of high energy astrophysics.

Since 2006 work on the extensive air shower (EAS) Cherenkov light array Tunka-133 with a geometric area of $1 \mathrm{~km}^{2}$ [1-3] has been carried out in Tunka Valley (50 km from Lake Baikal). Such an array will allow the investigation of cosmic rays in the energy range from $10^{15}$ to $10^{18} \mathrm{eV}$ by a uniform method. This energy range includes the knee in the energy spectrum at $3 \times 10^{15} \mathrm{eV}$ as well as other features of the spectrum probably connected to the transition from galactic to extragalactic cosmic rays.

The Tunka-133 array has been detecting Cherenkov light from EAS for the last two years, with a steady increase in the number of detectors (seven detectors from November 2006, 28 detectors from November 2007 and 84 detectors from November 2008) and the

\footnotetext{
* Corresponding author.

E-mail addresses: grss@api.isu.ru, grol08@rambler.ru (O.A. Gress).
}

array's effective area. The final array of 133 detectors was completed in October 2009 and the internal effective area reached $1 \mathrm{~km}^{2}$.

\section{Array description}

The Tunka-133 array consists of 133 optical detectors on the basis of photomultiplier EMI 9350 (20 cm photocathode diameter). The detectors are grouped into 19 clusters of seven detectors each. In a cluster one detector is located in the center, and six at a distance $85 \mathrm{~m}$ from the center in the vertices of a regular hexagon (Fig. 1). A container with control and data acquisition electronics (DAQ) is located near the central detector of each cluster. Each cluster electronics container is connected to the array DAQ center with a multi-wire cable consisting of four optical fibers and four copper wires.

For each optical detector (Fig. 2) the EAS Cherenkov light pulse shape is digitated by a Flash-ADC in the cluster electronics.

Measuring the atmospheric Cherenkov light provides the energy of the primary particle and the depth of the EAS maximum. The accuracy of the air shower core location is $\sim 6 \mathrm{~m}$, that of energy determination $\sim 15 \%$. The accuracy of the EAS maximum depth $X_{\text {max }}$ determination (from the Cherenkov light's lateral distribution steepness and pulse duration) is $25 \mathrm{~g} / \mathrm{cm}^{2}$. The energy threshold of the array is close to $10^{15} \mathrm{eV}$.

For one year of operation $(\sim 400 \mathrm{~h}$ ) the array will register more than 300 events with energies above $10^{17} \mathrm{eV}$ and with the EAS core position inside the array geometry. 


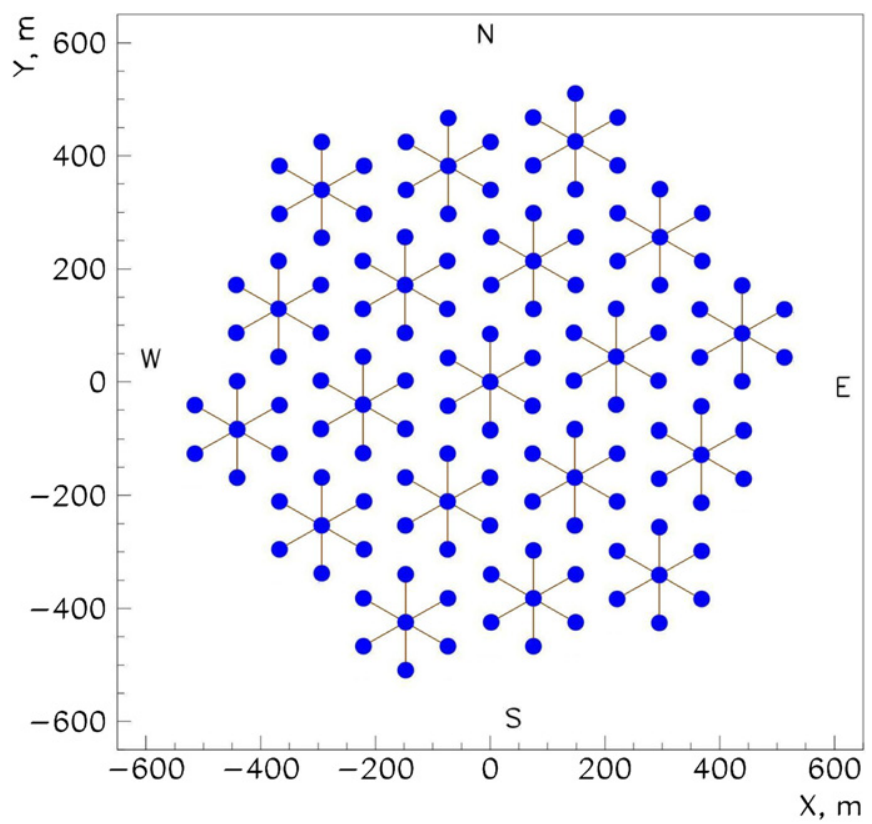

Fig. 1. Location of the optical detectors of the Tunka-133 installation. The detectors are grouped into 19 clusters of 7 detectors each. A container with DAQ is located near the central detector of each cluster.

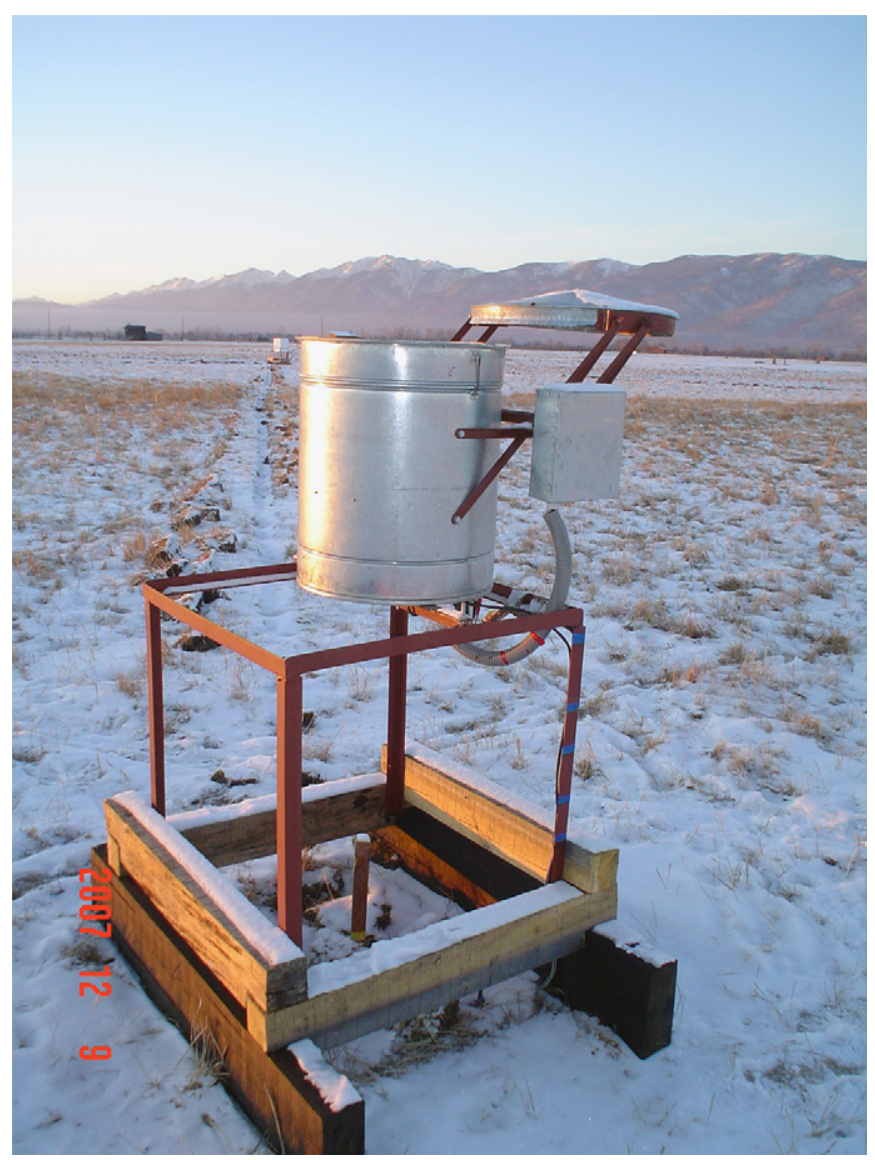

Fig. 2. Optical detector. The position of the detector lid is "open".

\section{Optical detector}

An optical detector consists of a metallic cylinder of $50 \mathrm{~cm}$ diameter containing a PMT (Fig. 2). The container window is directed towards the zenith and covered by plexiglass with heating

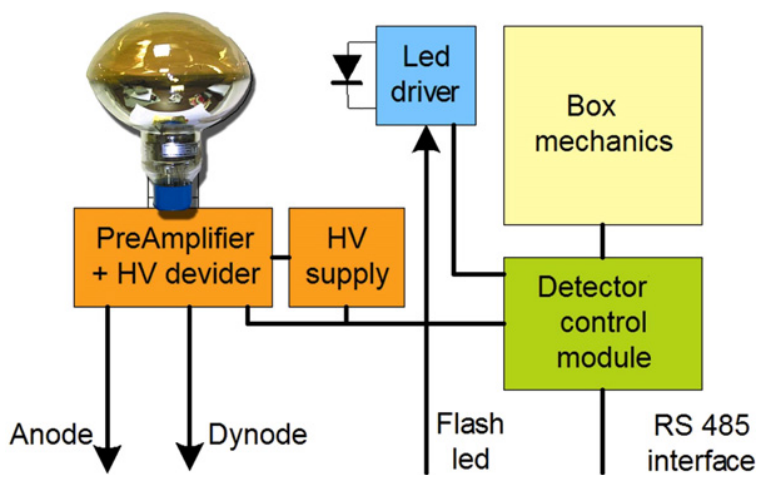

Fig. 3. Optical detector electronics.

against frost. The detector is equipped with a remotely controlled lid protecting the PMT from sunlight and precipitation. The detector efficiency is reduced steadily to about $80 \%$ of the vertical efficiency at a zenith angle of $40^{\circ}$ and to $50 \%$ at $50^{\circ}$.

The optical detector box contains a PMT with its high voltage supply, the preamplifiers for anode and dynode signals, a light emitting diode for both amplitude and time calibration and a controller (Fig. 3). The detector controller is connected with the cluster electronics via twisted pair by an RS-485 protocol.

To provide the necessary dynamic range of $10^{4}$, two analog signals, one from the anode and another from a dynode are read out. They are amplified and then transmitted to the central electronics hut of each cluster via 95 m RG-58 coaxial cables. The ratio of amplitudes of anode/dynode signals is $\sim 30$. Every PMT signal is digitized in the cluster box by an FADC.

The temperature range of the detector operation is from +20 to $-45{ }^{\circ} \mathrm{C}$.

\section{Data acquisition system}

The data acquisition system (DAQ) has three main components: (1) cluster electronics, (2) central DAQ (3) the software code which controls the acquisition of data from optical detectors.

\subsection{Cluster DAQ}

The cluster electronics consists of the cluster controller, four boards of four-channel FADCs, an adapter module connected to the optical-detector controller and separate temperature controller. All electronic modules (except the temperature controller) are in VME standard.

The FADC VME modules are designed on the basis of ADCs AD9430 and microchips FPGA XILINX Spartan XC3S200. Every FADC board (Fig. 4) consists of four simultaneously digitizing ADCs which are logically divided into two channels (one each for the anode and dynode signals from each optical detector). Each FADC has 12 bit resolution and samples at $200 \mathrm{MHz}$. The digitized signal from each ADC is transferred to the FPGA which handles the data. A double-buffered FIFO memory for 1024 counts was implemented inside the FPGA: while one buffer is waiting for readout, the second one is connected to the ADC outputs, thus minimizing the readout dead-time. Upon arrival of a trigger signal, 512 counts before and 512 after the trigger are read out.

The record of every event from a detector forms a row of 1024 amplitude measurements with a time step of 5 ns. Only one signal (anode) from each channel is connected to the comparator with digitally adjustable threshold, which produces the request signal to the trigger module (Fig. 4). The anode signal is also connected to an 


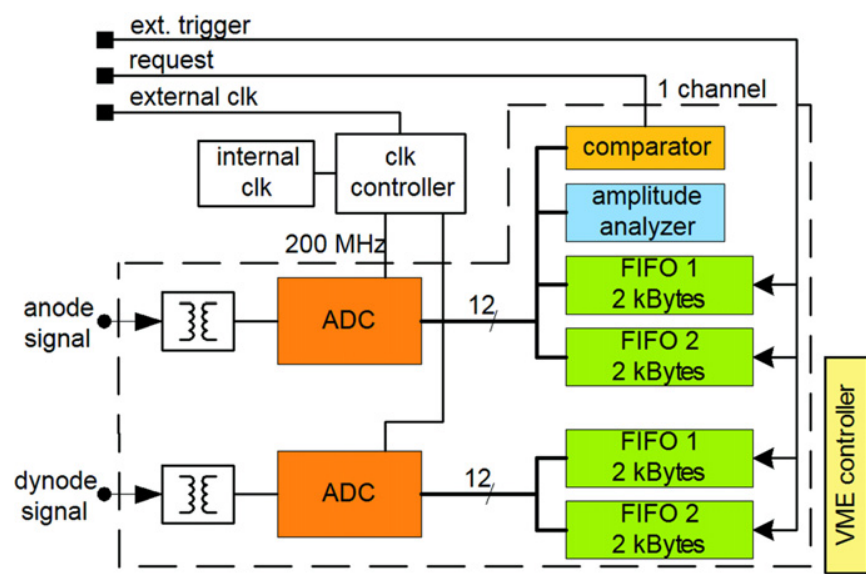

Fig. 4. Internal structure of FADC board (only two channels are shown; the other two are identical).

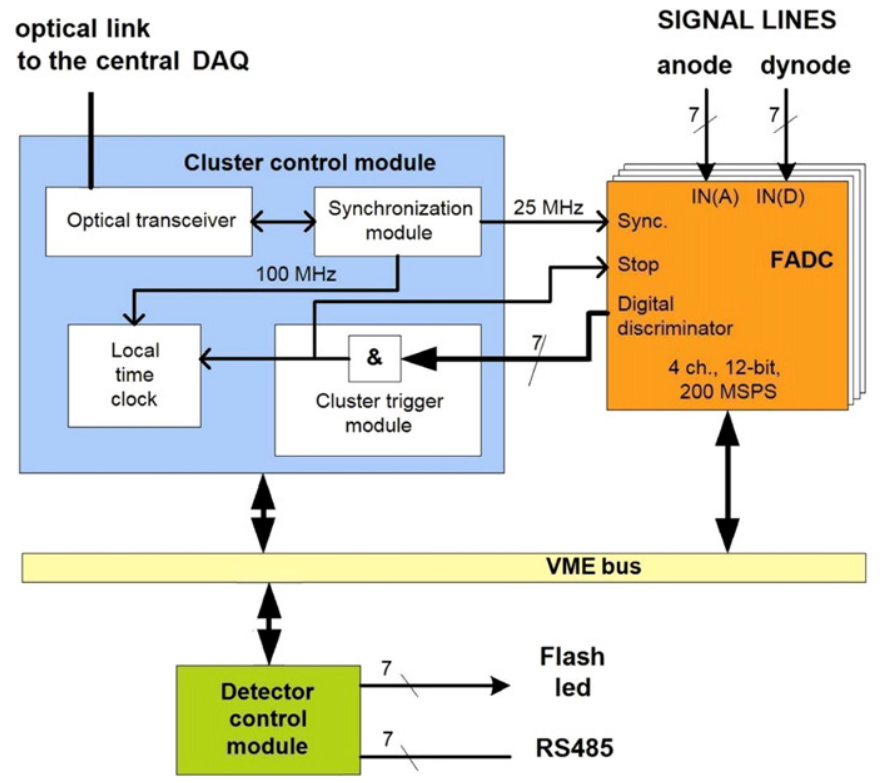

Fig. 5. Setup of the cluster electronics.

amplitude analyzer accumulating monitoring histograms for every channel.

The cluster controller (Fig. 5) contains the optical transceiver, synchronization module, local time clock and cluster trigger module.

The cluster trigger (local trigger) is formed by the coincidence of at least three pulses from optical detectors above threshold within a time window of $0.5 \mu \mathrm{s}$. The time of the local trigger is measured by the cluster clock.

The temperature controller switches on a heating element in the cluster box and as soon as the temperature rises up to $15^{\circ} \mathrm{C}$ it switches on the power for the VME crate. The temperature controller connects to the DAQ center via a radio channel based on XBee-PRO modules.

\subsection{Central DAQ}

Each cluster is connected to the DAQ center over a multi-wire cable containing four copper wires (for power supply voltages) and four optical fibers.
In the acquisition center the optical links are connected to five DAQ boards which are strictly synchronized among themselves at a frequency of $100 \mathrm{MHz}$ derived from a single oscillator. The DAQ board provides an interface for the four optical lines and is connected to the master PC by $100 \mathrm{Mbit} / \mathrm{s}$ Ethernet. Optical transceivers operating at $1 \mathrm{GHz}$ are responsible for the data transmission and for delivering $100-\mathrm{MHz}$ synchronization signals for the cluster clocks. So all cluster clocks operate at the same frequency.

The clusters are connected to the central DAQ via optical cables of unequal length, therefore the offsets between the cluster clocks must be calibrated. To measure the cable length and other factors influencing the propagation time the center sends a special packet to the cluster, which is returned by the cluster after decoding. The total time delay, divided by two, corresponds to the propagation delay. Then the center sends a timer reset command simultaneously to all cluster clocks. The knowledge of the propagation delay allows to calculate the time offsets between the cluster clocks. The accuracy of this time synchronization is about $10 \mathrm{~ns}$. To avoid any mis-timing, the synchronization is being checked by hardware once per second.

\section{Preliminary results and plans}

The whole Tunka-133 array started data taking at the end of October 2009. Now (at the end of the February 2010) we have data from nearly $300 \mathrm{~h}$ of observation during clear moonless nights. The average trigger rate was about $2 \mathrm{~Hz}$ (Fig. 6).

Fig. 7 shows the distribution of the number of clusters giving local triggers in one physical event. During the one night there are 3-10 events in which all 19 clusters give a local trigger. In such events practically all 133 optical detectors are hit. The energy of such events are close to or more than $10^{17} \mathrm{eV}$.

An example of Cherenkov light pulses at two detectors of one cluster is shown in Fig. 8. For this event the core position of the EAS is at a distance of about $700 \mathrm{~m}$ from these detectors and the full width at half-maximum $F W H M_{i}$ is about $150 \mathrm{~ns}$, in good agreement with the expected value.

The low energy part of the spectrum is shown in Fig. 9 together with the Tunka- 25 spectrum. The energy of $100 \%$ efficiency is about $2 \times 10^{15} \mathrm{eV}$. It will be possible to decrease the energy threshold of the array by selecting showers with core position located inside the clusters' geometric area.

To increase the effective up-time of the array, we plan to upgrade the Cherenkov array with additional detectors which may register EAS during the day time: antennas for registering radio-pulses from EAS and scintillation counters for detecting muons and electrons [4].

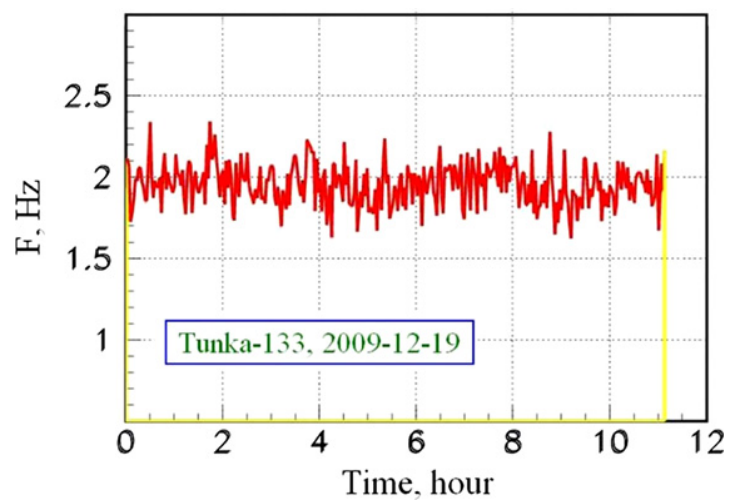

Fig. 6. Trigger rate during one night. 


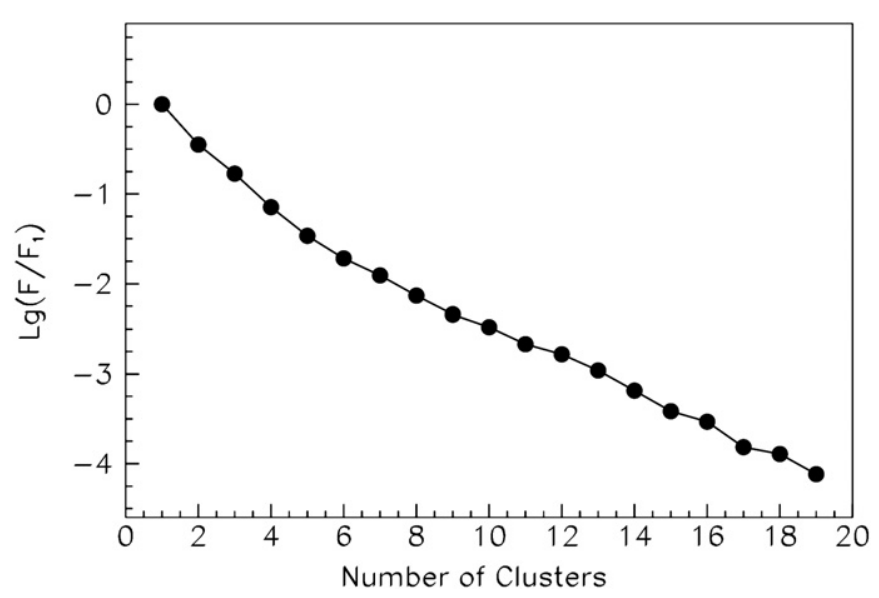

Fig. 7. Distribution of the number of clusters participating in one event.

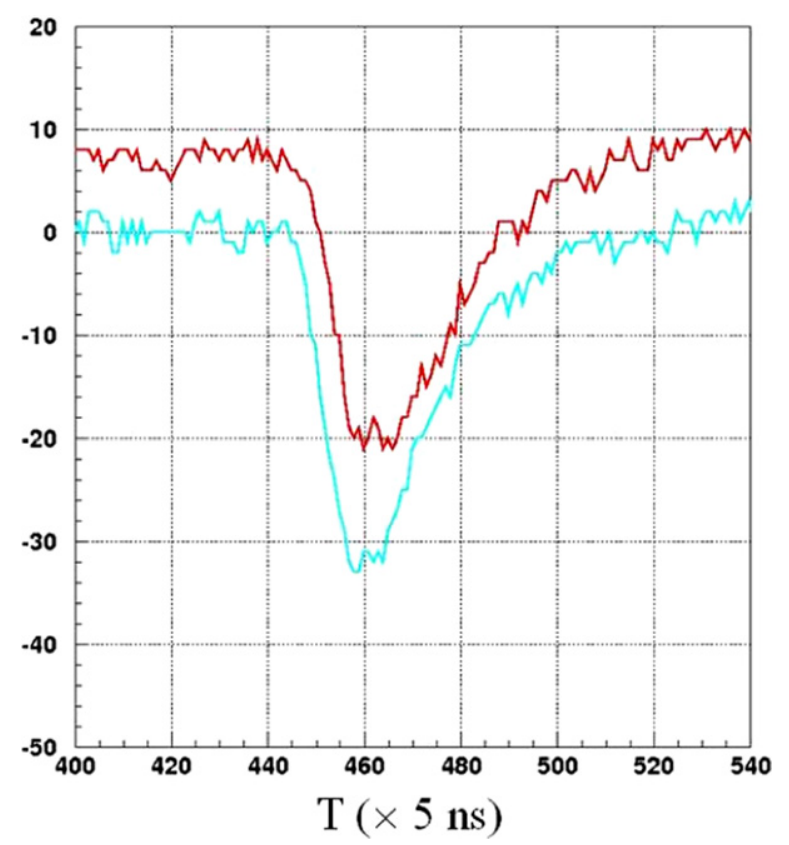

Fig. 8. Cherenkov light pulses at two detectors of one cluster. The distance from the EAS core is about $700 \mathrm{~m}$.

The registration of the radio flux from EAS is - like the registration of Cherenkov light - a calorimetric method which may give information about the energy of primary particles and the depth of the shower maximum with good accuracy. To cross-check this method, we plan to install a network of 20 radio antennas for common operation with the Cherenkov array. The first three antennas were installed in 2008 and 2009 for tests.

The deployment of scintillation counters within the Tunka array would provide a cross-calibration of different methods of EAS parameter measurements since all components will be recorded simultaneously. It has been shown [5] that by using 20 muon detectors of $10 \mathrm{~m}^{2}$ area placed inside the geometric area of Tunka-

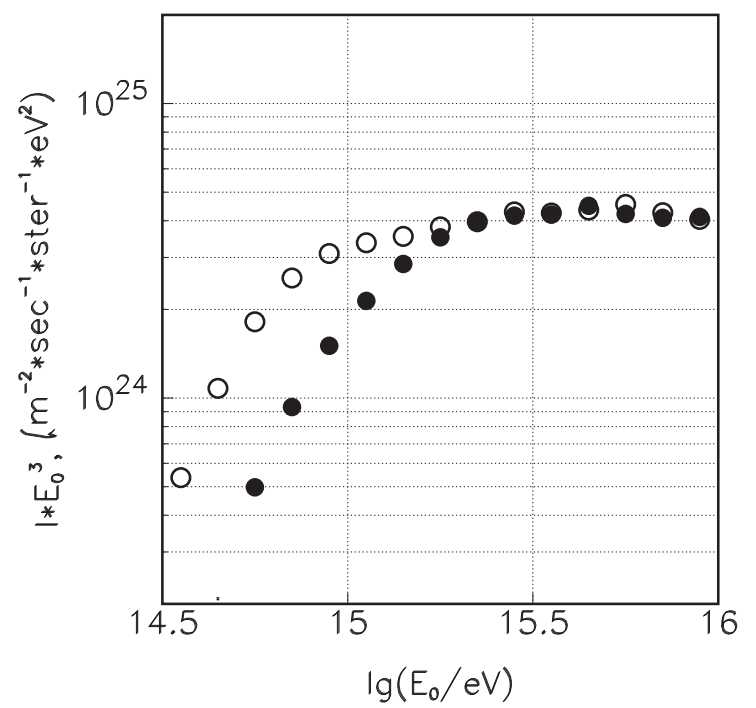

Fig. 9. The energy spectrum from Tunka-25 (open circles) and Tunka-133 (filled circles) in the threshold region.

133 it is possible to reach an accuracy of about $10 \%$ in the total muon number at $10^{17} \mathrm{eV}$.

\section{Conclusion}

Since October 2009 the Tunka-133 has been taking data. The first results show that the array parameters are in good agreement with expected values. Recording the pulse shape for each detector has proved to be the most reliable measurement of $X_{\max }$ and opens the possibility of reconstructing EAS with the core outside the array geometry [6]. Work has been started for upgrading Tunka-133 with day-time operating detectors (radio-antennas and muon scintillation counters).

\section{Acknowledgments}

The present work is supported by the Russian Ministry of Education and Science, program "Development of Scientific Potential of High Energy Schools" (projects 2.2.1.1/1483, 2.1.1/1539, 2.2.1/5901) and by the Russian Foundation of Basic Research (Grant 09-02-1000, 09-02-12287).

\section{References}

[1] N.M. Budnev, et al., (Tunka Collaboration), in: Proceedings of 29th ICRC, Pune, India, 2005, arXiv:astro-ph/0511229.

[2] N.M. Budnev, et al., (Tunka Collaboration), in: Proceedings of 30th ICRC, Merida, Yucatan, Mexico, 2007, arXiv:0801.3037.

[3] N.M. Budnev, et al., (Tunka Collaboration), in: Proceedings of 29th ICRC, Pune, India, vol. 6, 2005, p. 257, arXiv: astro-ph/0511220.

[4] N.M. Budnev, et al., (Tunka Collaboration), in: Proceedings of 31st ICRC, Lodz, Poland, 2009, ID=1069, arXiv:1003.0089.

[5] N.N. Kalmykov, et al., in: Proceedings of 31st ICRC, Lodz, Poland, ID $=1073$

[6] E.E. Korosteleva, et al., in: Proceedings of 31st ICRC, Lodz, Poland, 2009, ID =492. 